\title{
Uric Acid Contributes Greatly to Hepatic Antioxidant Capacity Besides Protein
}

\author{
T. MIKAMI ${ }^{1}$, M. SORIMACHI ${ }^{2}$ \\ ${ }^{1}$ Department of Health and Sports Science, Nippon Medical School, Musashino, Tokyo, Japan, \\ ${ }^{2}$ JOKAKU Inc., Minato-ku, Tokyo, Japan
}

Received November 15, 2016

Accepted May 2, 2017

On-line September 22, 2017

\section{Summary}

Uric acid is the end-product of purine nucleotide metabolism and an increase in uric acid concentration in the body results in hyperuricemia, ultimately leading to gout. However, uric acid is a potent antioxidant and interacts with reactive oxygen species (ROS) to be non-enzymatically converted to allantoin. Uric acid accounts for approximately $60 \%$ of antioxidant capacity in the plasma; however, its contribution to tissue antioxidant capacity is unknown. In this study, the contribution of uric acid to tissue antioxidant capacity and its conversion to allantoin by scavenging ROS in tissue were examined. The results showed that a decrease in hepatic uric acid content via allopurinol administration significantly reduced hepatic total-radical trapping antioxidant parameter (TRAP) content in protein-free cytosol. Additionally, treating protein-free cytosol with uricase led to a further reduction of hepatic TRAP content. Allantoin was also detected in the solution containing protein-free cytosol that reacted with ROS. These findings suggest that in the absence of protein, uric acid contributes greatly to antioxidant capacity in the liver, where uric acid is converted to allantoin by scavenging ROS.

\section{Key words}

Uric acid - Antioxidant - Total-radical trapping antioxidant parameter • Allantoin

\section{Corresponding author}

T. Mikami, Department of Health and Sports Science, Nippon Medical School, 1-7-1 Kyounan-cho, Musashino, Tokyo 180-0023, Japan. Fax: +81-422-34-3451. E-mail: mikami@nms.ac.jp

\section{Introduction}

As humans lack the uricase enzyme, which oxidizes uric acid to allantoin, the end-product of purine nucleotide degradation in man is uric acid. An increase in uric acid content in the body results in hyperuricemia and gout. Hyperuricemia is also a risk factor for arteriosclerosis, diabetes, and renal failure. Therefore, high level of uric acid is considered as harmful. On the other hand, uric acid has a potent capacity to scavenge reactive oxygen species (ROS) and this ability is closely comparable to that of vitamin C (Ames et al. 1981). In addition, uric acid concentration in plasma is approximately $15-450 \mu \mathrm{M}$, which is 10 times higher than that of ascorbic acid (Ames et al. 1981), showing that uric acid is a potent radical scavenger in plasma.

Total-radical trapping antioxidant parameter (TRAP) represents the capacity that drugs, natural substances and biological samples scavenge active free oxygen radicals (Alanko et al. 1993). In plasma, uric acid concentration correlates with the plasma TRAP concentration (Nalsen et al. 2006). Patients with rheumatism (Grootveld and Halliwell 1987), Down's syndrome (Zitnanova et al. 2004), and Parkinson's disease (Schwarzschild et al. 2008) have a significantly lower concentration of serum or plasma uric acid than healthy individuals. This is because uric acid is degraded by ROS, whose levels increase in these conditions. Thus, uric acid greatly contributes to plasma antioxidant capacity. With regard to tissue antioxidant capacity, Evelson reported that protein contributes to $60-85 \%$ of TRAP in tissue (liver, brain, kidney, and heart) 
homogenate (Evelson et al. 2001). Judging from a contribution of uric acid to plasma antioxidant capacity, it is expected that uric acid would also contribute to antioxidant capacity in the intracellular environment of the cell. However, no previous study showed contribution of uric acid to antioxidant capacity in the intracellular fluid.

When uric acid reacts with ROS, uric acid is non-enzymatically converted to allantoin (Plank et al. 2011). Patients with rheumatism (Grootveld and Halliwell 1987) and Down's syndrome (Zitnanova et al. 2004) have significantly increased concentrations of serum or plasma allantoin. However, no study to date has shown the oxidization of uric acid to allantoin by ROS as antioxidant in tissues.

In the present study, we examined the contribution of uric acid to antioxidant capacity in the intracellular fluid by comparing TRAP content of hepatic protein-free cytosol between normal and allopurinoltreated mice. Additionally, we examined whether uric acid is non-enzymatically converted to allantoin by ROS in tissue or not.

\section{Materials and Methods}

\section{Animals and drug treatment}

The Animal Care and Use Committee of Nippon Medical School approved all experimental and animal care procedures. A total of 16, 8-week-old, Crl:CD1 mouse (ICR mice) were randomly divided into two groups, which were control and allopurinol-treated mice. Allopurinol is inhibitor for xanthine oxidase that catalyzes xanthine and hypoxanthine to uric acid. Therefore, allopurinol administration results in decrease of uric acid content in tissues. Control mice were fed with a commercial mouse powder diet (Oriental Yeast Co., Tokyo, Japan), whereas allopurinol-treated mice were fed with the powder diet containing $0.01 \mathrm{~g}$ allopurinol per $100 \mathrm{~g}$ food. Both groups consumed approximately $5 \mathrm{~g}$ food daily for 8 days. On day 9 , the mice were scarified by bleeding via abdominal aorta under anesthesia. Immediately, the liver was washed with saline, frozen in liquid nitrogen, and stored at $-80^{\circ} \mathrm{C}$ until analysis. Blood was collected and centrifuged at $1,000 \times \mathrm{g}$ for $20 \mathrm{~min}$. Plasma was stored at $-80{ }^{\circ} \mathrm{C}$ until analysis.

The liver was vigorously homogenized in $100 \mathrm{mM}$ Tri-HCl buffer ( $\mathrm{pH} 7.4,10$ times the volume of tissue) using a hand homogenizer. A part of the homogenate was used to analyze thiobarbituric acid reactive substance (TBARS) as previously described by Kosugi et al. (1993). The rest of the homogenate was centrifuged at $750 \times \mathrm{g}$ for $10 \mathrm{~min}$. A portion of the supernatant was used to analyze superoxide dismutase (SOD) activity as previously described by Spitz and Oberley (1989). The remainder of the supernatant was re-centrifuged at $100,000 \times \mathrm{g}$ for $90 \mathrm{~min}$ to obtain the cytosolic fraction. Cytosol was filtered using an ultrafree MC filter (Merck Millipore, USA, pore size $=3,000 \mathrm{~A}$ ) to remove protein from the cytosolic fraction.

\section{TRAP analysis}

Protein-free cytosol was used to measure TRAP content as previously described by Alanko et al. (1993). Briefly, $0.45 \mathrm{ml}$ of $0.1 \mathrm{M}$ phosphate-buffered saline (pH 7.4), $0.05 \mathrm{ml}$ of $0.5 \mathrm{mg} / \mathrm{ml} \mathrm{luminol}$, and $0.02 \mathrm{ml}$ of $120 \mathrm{mM}$ linoleic acid were mixed in a cuvette. The cuvette was placed in a temperature-controlled sample carousel of a Lumi Counter-2500 (Microtec Co., Tokyo, Japan) and incubated at $37^{\circ} \mathrm{C}$ for $5 \mathrm{~min}$. The assay was

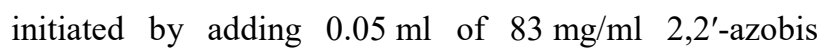
(2-amidinopropane) dihydrochloride (ABAP). After the chemiluminescence in the cuvette increased and became stable at $37^{\circ} \mathrm{C}, 0.02 \mathrm{ml}$ protein-free cytosol or standard was added to the cuvette and the time when chemiluminescence stopped was recorded and defined as the peroxyl radical trapping capacity. Water-soluble tocopherol, Trolox (Aldrich, USA), which is known to trap two radicals per molecule, was used as standard.

\section{HPLC analysis}

To examine whether the uric acid present in the protein-free cytosol was converted to allantoin by reaction with ROS, the reaction mixture used for TRAP analysis was analyzed for allantoin using highperformance liquid chromatography (HPLC) as previously described by Grootveld and Halliwell (1987) and Lagendijk et al. (1995), with modifications (Mikami et al. 2000a). The concentration of purines (urate, hypoxanthine, and xanthine), ascorbate, $\alpha$-tocopherol, and glutathione (GSH) in protein-free cytosol was measured using HPLC as previously described by Itakura et al. (1981), Otsuka et al. (1986), Milne and Botnen (1986), and Takanuki et al. (1988), respectively.

\section{Statistics}

The results are expressed as the mean \pm SE. The statistical significance of differences between two groups 
was tested using an unpaired two-tailed t-test. Analysis of variance (ANOVA) and Scheffe's test were used to determine the significance between more than two groups. Differences were considered statistically significant at $\mathrm{p}<0.05$.

\section{Results}

To reduce uric acid content in the body, mice were fed with a diet containing allopurinol for 8 days.
Consumption of allopurinol-containing food resulted in a significant decrease of uric acid in the plasma (Table 1) and protein-free hepatic cytosol (Fig. 1). On the contrary, the sum of hypoxanthine and xanthine in protein-free hepatic cytosol was significantly increased by allopurinol consumption (Table 1). These findings suggest that consumption of allopurinol-containing food inhibited uric acid formation and led to a significant decrease in uric acid content in the liver.

Table 1. Effects of allopurinol administration on the concentration of uric acid in plasma, the content of purines (hypoxanthine and xanthine), TBARS, and the activity of SOD in liver.

\begin{tabular}{lcccc}
\hline & \multicolumn{2}{c}{ Llasma } & TBARS & SOD \\
\cline { 2 - 5 } & $\begin{array}{c}\text { Uric acid } \\
\boldsymbol{\mu m o l} / \mathbf{l}\end{array}$ & $\begin{array}{c}\text { Hypo }+ \text { Xan } \\
\boldsymbol{\mu m o l} / \mathbf{g} \text { tissue }\end{array}$ & $\begin{array}{c}\boldsymbol{\mu} \mathbf{m o l} / \mathbf{g} \text { tissue } \\
\text { U/mg protein }\end{array}$ \\
\hline $\begin{array}{l}\text { Control } \\
\text { Allopurinol }\end{array}$ & $199 \pm 20$ & $838 \pm 65$ & $6.4 \pm 0.2$ & $488 \pm 55$ \\
\hline
\end{tabular}

Values are mean \pm SEM. $n=8$. Hypo + Xan, sum of hypoxanthine and xanthine. $* \mathrm{p}<0.05$ vs. control.

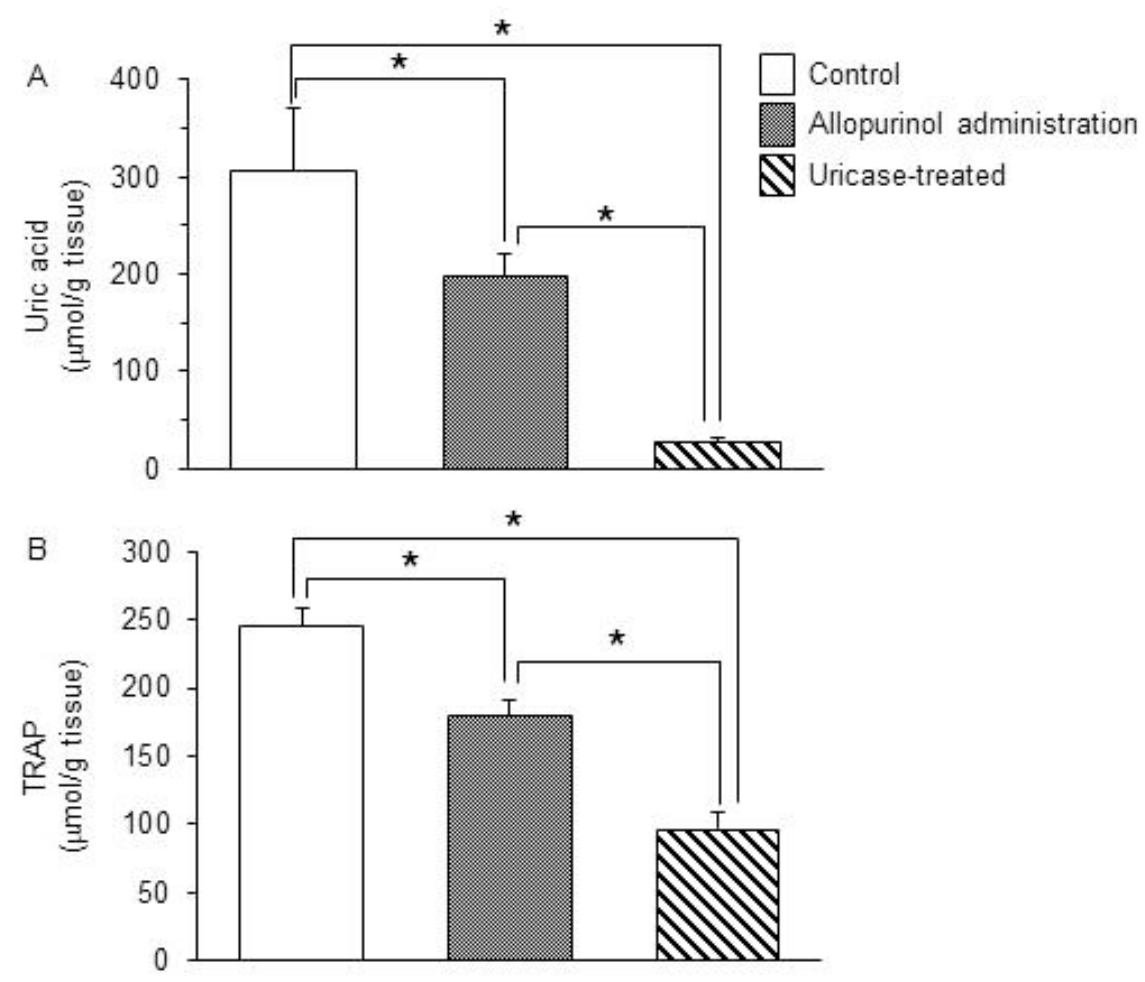

Fig. 1. Uric acid (A) and TRAP (B) levels in protein-free hepatic cytosol. Protein-free hepatic cytosol of control and allopurinol-administered mice was analyzed. In addition, protein-free hepatic cytosol of control mice was treated with uricase and analyzed. Values are expressed as the mean \pm SEM $(n=8) . \quad * p<0.05$, significant difference between two groups.

Next, to investigate whether the change in uric acid content of protein-free hepatic cytosol influences antioxidant capacity, TRAP content in protein-free cytosol of liver was compared between control and allopurinol-fed mice. TRAP content in allopurinol-fed mice significantly decreased to $70 \%$ of that in control mice (Fig. 1). To examine the effect of other watersoluble antioxidants on the decrease in hepatic TRAP, the content of ascorbate and GSH in protein-free hepatic cytosol was measured. The level of ascorbate and GSH in 
protein-free hepatic cytosol was almost the same in both, control and allopurinol-fed mice (Table 1). On the contrary, both TBARS content and SOD activity were significantly higher in allopurinol-fed mice than those in control mice $(\mathrm{p}<0.05)$ (Table 1). Both TBARS and SOD activity would be attributed to hypoxanthine induced by allopurinol treatment (Mesquita Casagrande et al. 2013). However, there is possibility that the increase of SOD activity in allopurinol-fed mice could be to compensate for the reduction of antioxidant capacity due to the decrease of uric acid content.

To assess whether a further decrease of uric acid content in protein-free cytosol influenced antioxidant capacity in tissue, the protein-free hepatic cytosol from control mice was treated with uricase to reduce uric acid and analyzed for TRAP content. After uricase treatment, TRAP level in protein-free hepatic cytosol decreased significantly, to $25 \%$ of pretreatment level with a considerable decrease in uric acid level (Fig. 1). To assess the influence of uricase treatment on other watersoluble antioxidants, we determined the concentration of ascorbate and GSH in uricase-treated hepatic cytosol using HPLC analysis. Ascorbate and GSH levels were almost the same in the protein-free hepatic cytosol regardless of uricase treatment (Fig. 2).

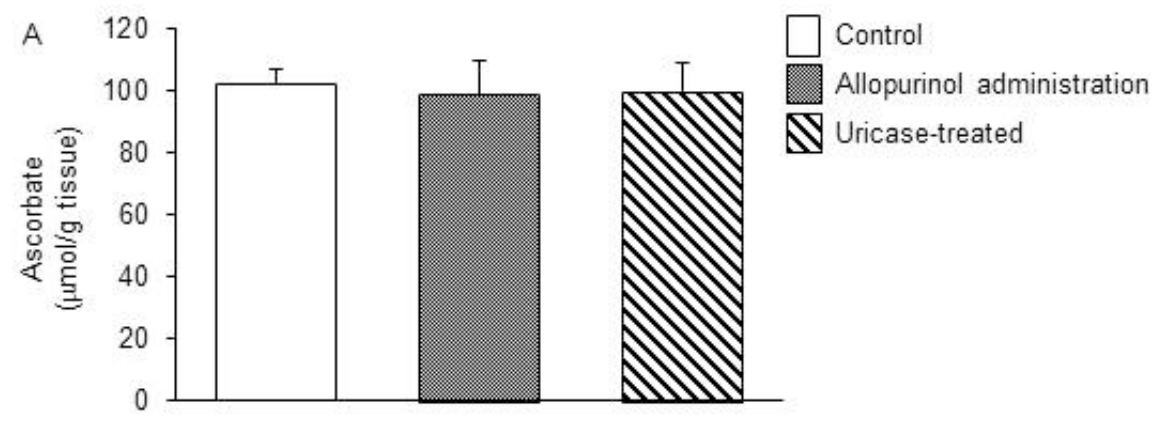

Fig. 2. Ascorbate (A) and GSH (B) levels in protein-free hepatic cytosol. Protein-free hepatic cytosol of control and allopurinol-administered mice was analyzed. In addition, protein-free hepatic cytosol of control mice was treated with uricase and analyzed. Values are expressed as the mean \pm $\operatorname{SEM}(n=8)$.

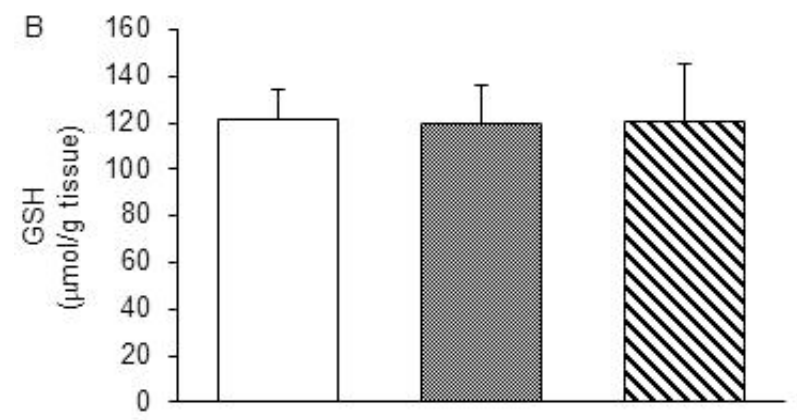

Thus, to assess whether uric acid-containing protein-free hepatic cytosol is indeed converted to allantoin by reacting with ROS, the reaction solution used for TRAP analysis was also analyzed via HPLC for allantoin. Addition of ABAP, which triggers ROS formation, resulted in a significant increase of allantoin concentration in the reaction solution containing proteinfree hepatic cytosol from control mice, compared with that without addition of ABAP (Figs 3A and 3B). Furthermore, to confirm that uric acid is indeed converted to allantoin in the present TRAP assay, the reaction solution containing uric acid with or without addition of ABAP was also analyzed via HPLC for allantoin. When uric acid was added into the reaction mixture of TRAP assay instead of hepatic protein-free cytosol, clear peak of

allantion was observed with addition of ABAP (Figs 3C and 3D).

\section{Discussion}

In this study, to examine contribution of uric acid to hepatic antioxidant capacity in mice, the level of TRAP in protein-free hepatic cytosol containing different levels of uric acid was compared. In protein-free hepatic cytosol, the decrease of uric acid via allopurinol administration resulted in significant suppression of TRAP. Additionally, uricase treatment led to further decrease of hepatic TRAP. However, neither allopurinol administration nor uricase treatment changed the hepatic content of ascorbate and GSH. Allantoin was detected in 
the reaction solution following the measurement of TRAP. These findings suggested that uric acid contributes greatly to antioxidant capacity in the liver and it is converted to allantoin by scavenging ROS.
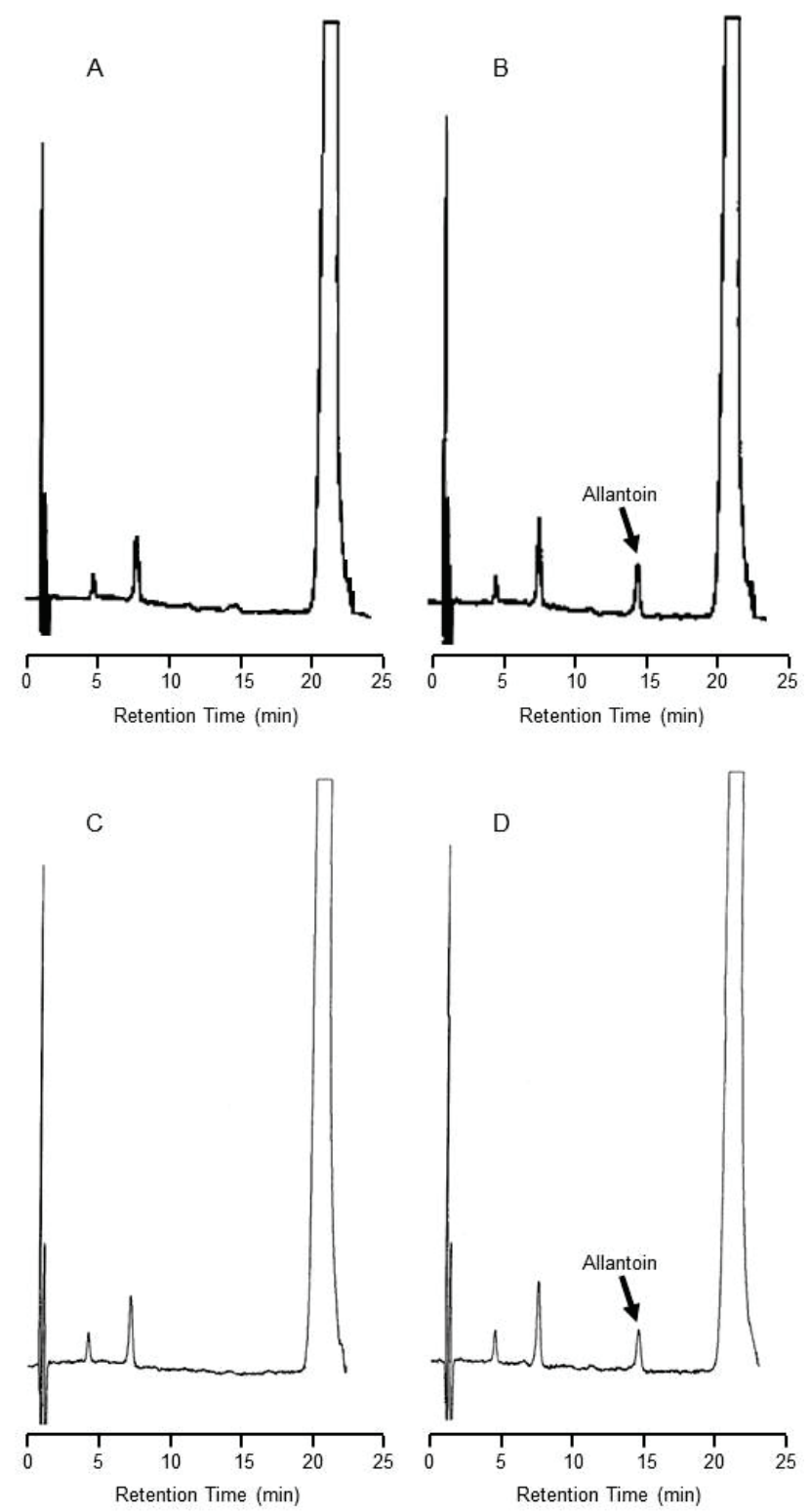

Fig. 3. A representative HPLC chromatogram for allantoin in protein-free hepatic cytosol and uric acid. $(\mathbf{A}, \mathbf{C})$ Before addition of ABAP, (B, D) after addition of ABAP. Addition of ABAP in the TRAP analysis resulted in appearance of an allantoin peak.

Uric acid has a strong antioxidant capacity, equal to ascorbic acid (Ames et al. 1981). A strong relationship has been shown between uric acid concentration and TRAP level in human plasma (Nalsen et al. 2006). Our previous findings where plasma uric acid concentration in human subjects changed after oral administration of uricosuric drug (benzbromarone) or uric acid precursor (IMP) also showed that plasma TRAP level has a significantly positive correlation with that of plasma uric acid (Mikami et al. 2000b). This may be attributed to the fact that uric acid has higher plasma concentration than other major antioxidants (ascorbate, $\mathrm{GSH}$, and $\alpha$-tocopherol). These findings suggest that uric acid contributes greatly to plasma antioxidant capacity.

On the other hand, all tissues contain antioxidant enzymes (such as SOD, catalase, glutathione peroxidase). Especially, SOD exhibits a strong capacity to scavenge superoxide, therefore, proteins containing antioxidant enzymes account for the majority of antioxidant capacity in tissues. Evelson et al. (2001) showed that protein contributes to $60-85 \%$ of TRAP in tissue (liver, brain, kidney, and heart) homogenate. Therefore, to examine contribution of uric acid to tissue antioxidant capacity in the absence of proteins, we compared TRAP content in hepatic protein-free cytosol between control and allopurinol-fed mice. Allopurinol consumption decreased hepatic TRAP to $80 \%$ of that in control with a significant decrease of uric acid content (Fig. 1). However, allopurinol cannot completely inhibit xanthine oxidase activity, therefore, low level of uric acid remained in the protein-free cytosol of allopurinol-fed mice. To further reduce uric acid content, the protein-free cytosol from control mice was treated with uricase, which metabolizes uric acid to allantoin, and the resultant mixture was used to measure TRAP. Uricase treatment significantly decreased TRAP content by $40 \%$ in untreated cytosol (Fig. 1), with no change in the content of other major water-soluble antioxidants, ascorbate, and GSH (Fig. 2). These findings suggest that uric acid contributes greatly to hepatic antioxidant capacity, besides proteins. To our knowledge, this is the first report describing the significant contribution of uric acid to scavenging ROS in the liver.

As humans lack uricase enzyme, uric acid is the end-product of purine metabolism in the body. However, when uric acid reacts with ROS as an antioxidant, uric acid is further metabolized to allantoin (Plank et al. 2011). Patients with rheumatism (Grootveld and Halliwell 1987) and Down's syndrome (Zitnanova et al. 2004) have significantly higher concentration of serum or plasma allantoin because ROS production is increased in these conditions. Furthermore, individuals performing intense exercise show an increase of plasma allantoin concentration because intense exercise results in increased ROS formation (Mikami et al. 2000a). To confirm allantoin formation by reaction of uric acid with 
ROS, the reaction solution used for TRAP analysis was also analyzed via HPLC for allantoin (Figs 3A and 3B). Additionally, when uric acid was added to the reaction mixture of TRAP assay instead of hepatic protein-free cytosol, clear allantoin peak was observed with addition of ABAP (Figs 3C and 3D). These findings suggest that uric acid is indeed converted to allantoin by reacting with ROS in tissues.

In this study, we used mice since rodents produce uricase, which converts uric acid to allantoin. That is probably the reason for uric acid content in the liver of mice being lower than that observed in man. If the results observed in mice in this study are applied to man, hepatic TRAP content may be much higher in healthy individuals due to higher uric acid content, whereas TRAP content may be considerably reduced by allopurinol administration and uricase treatment. Therefore, it is envisioned that contribution of uric acid to hepatic antioxidant capacity besides proteins would be much higher than that currently observed in man.

Recently, antioxidant property of uric acid has been applied to the treatment of diseases. For example, onset of Parkinson's disease is attributed to the increase in ROS production. Meanwhile, a decrease in serum uric acid concentration is also seen in patients with Parkinson's disease (Schwarzschild et al. 2008). Therefore, to increase uric acid content in the body and enhance antioxidant capacity, inosine, which is metabolized to uric acid, was orally administered to the patients of Parkinson disease in a previous study, which showed that oral administration of inosine can retard the progress of Parkinson's disease (Schwarzschild et al. 2008). Furthermore, inosine promotes the development of primarily cultured neurons under stressful conditions and prevents onset of depressive behavior in mice (Muto et al. 2014). The reason for inosine exerting an antidepressant effect is not clear; probably the antioxidant capacity of uric acid metabolized from inosine contributes to antidepressant effect of inosine. In the future, antioxidant capacity of uric acid can be utilized for treatment of a wide range of diseases that are caused by oxidative stress.

\section{Conclusion}

The contribution of uric acid to tissue antioxidant capacity and its conversion to allantoin by scavenging ROS in tissue were examined. A decrease in hepatic uric acid content via allopurinol administration significantly reduced hepatic TRAP content in proteinfree cytosol. Allantoin was also detected in the solution containing protein-free cytosol that reacted with ROS. These findings suggest that in the absence of protein, uric acid contributes greatly to antioxidant capacity in the liver, where uric acid is converted to allantoin by scavenging ROS. The antioxidant capacity of uric acid might be useful for treatment of diseases. In the future, enhancement of the antioxidant capacity of uric acid via inosine administration might be utilized for treatment of diseases that in which pathology oxidative stress play a role.

\section{Conflict of Interest}

There is no conflict of interest.

\section{Acknowledgements}

This study was supported by a Grant-in-Aid for Scientific Research for 2010 and 2011 from The Ministry of Education, Science, Sports, and Culture of Japan.

\section{References}

ALANKO J, RIUTTA A, MUCHA I, VAPAATALO H, METSA-KETELA T: Modulation of arachidonic acid metabolism by phenols: relation to positions of hydroxyl groups and peroxyl radical scavenging properties. Free Radic Biol Med 14: 19-25, 1993.

AMES BN, CATHCART R, SCHWIERS E, HOCHSTEIN P: Uric acid provides an antioxidant defense in humans against oxidant- and radical-caused aging and cancer: a hypothesis. Proc Natl Acad Sci U S A 78: 6858-6862, 1981.

EVELSON P, TRAVACIO M, REPETTO M, ESCOBAR J, LLESUY S, LISSI EA: Evaluation of total reactive antioxidant potential (TRAP) of tissue homogenates and their cytosols. Arch Biochem Biophys 388: 261-266, 2001.

GROOTVELD M, HALLIWELL B: Measurement of allantoin and uric acid in human body fluids. A potential index of free-radical reactions in vivo? Biochem $J$ 243: 803-808, 1987. 
ITAKURA M, SABINA RL, HEALD PW, HOLMES EW: Basis for the control of purine biosynthesis by purine ribonucleotides. J Clin Invest 67: 994-1002, 1981.

KOSUGI H, KOJIMA T, KIKUGAWA K: Characteristics of the thiobarbituric acid reactivity of human urine as a possible consequence of lipid peroxidation. Lipids 28: 337-343, 1993.

LAGENDIJK J, UBBINK JB, VERMAAK WJ: The determination of allantoin, a possible indicator of oxidant status, in human plasma. J Chromatogr Sci 33: 186-193, 1995.

MESQUITA CASAGRANDE AC, WAMSER MN, DE LIMA DD, PEREIRA DA CRUZ JG, WYSE AT, DAL MAGRO DD: In vitro stimulation of oxidative stress by hypoxanthine in blood of rats: prevention by vitamins E plus C and allopurinol. Nucleosides Nucleotides Nucleic Acids 32: 42-57, 2013.

MIKAMI T, KITA K, TOMITA S, QU GJ, TASAKI Y, ITO A: Is allantoin in serum and urine a useful indicator of exercise-induced oxidative stress in humans? Free Radic Res 32: 235-244, 2000a.

MIKAMI T, YOSHINO Y, ITO A: Does a relationship exist between the urate pool in the body and lipid peroxidation during exercise? Free Radic Res 32: 31-39, $2000 \mathrm{~b}$.

MILNE DB, BOTNEN J: Retinol, alpha-tocopherol, lycopene, and alpha- and beta-carotene simultaneously determined in plasma by isocratic liquid chromatography. Clin Chem 32: 874-876, 1986.

MUTO J, LEE H, LEE H, UWAYA A, PARK J, NAKAJIMA S, NAGATA K, OHNO M, OHSAWA I, MIKAMI T: Oral administration of inosine produces antidepressant-like effects in mice. Sci Rep 4: 4199, 2014.

NALSEN C, OHRVALL M, KAMAL-ELDIN A, VESSBY B: Plasma antioxidant capacity among middle-aged men: the contribution of uric acid. Scand J Clin Lab Invest 66: 239-248, 2006.

OTSUKA M, KURATA T, ARAKAWA N: Antiscorbutic effect of dehydro-L-ascorbic acid in vitamin C-deficient guinea pigs. J Nutr Sci Vitaminol (Tokyo) 32: 183-190, 1986.

PLANK MS, CALDERON TC, ASMEROM Y, BOSKOVIC DS, ANGELES DM: Biochemical measurement of neonatal hypoxia. J Vis Exp 54: 2948, 2011.

SCHWARZSCHILD MA, SCHWID SR, MAREK K, WATTS A, LANG AE, OAKES D, SHOULSON I, ASCHERIO A, HYSON C, GORBOLD E, RUDOLPH A, KIEBURTZ K, FAHN S, GAUGER L, GOETZ C, SEIBYL J, FORREST M, ONDRASIK J: Serum urate as a predictor of clinical and radiographic progression in Parkinson disease. Arch Neurol 65: 716-723, 2008.

SPITZ DR, OBERLEY LW: An assay for superoxide dismutase activity in mammalian tissue homogenates. Anal Biochem 179: 8-18, 1989.

TAKANUKI K, IGARASHI T, HATA K, HORI H, SHIBATA T, KITAGAWA H, SATOH T, INAYAMA S: Glutathione depletion by 2-nitroimidazole-1-acetohydroxamic acid as a radiosensitizer in hypoxic rat hepatocytes. Biochem Int 17: 155-162, 1988.

ZITNANOVA I, KORYTAR P, ARUOMA OI, SUSTROVA M, GARAIOVA I, MUCHOVA J, KALNOVICOVA T, PUESCHEL S, DURACKOVA Z: Uric acid and allantoin levels in Down syndrome: antioxidant and oxidative stress mechanisms? Clin Chim Acta 341: 139-146, 2004. 